\title{
An Alternative Method to Reconstruct Severely Atrophic Mandible: Case Report
}

\author{
Gaffrée Gustavo* \\ Director of Surgical Department, Lourenço Jorge Hospital, Brazil
}

Received: November 10, 2017; Published: November 16, 2017

*Corresponding author: Gaffrée Gustavo, Director of Surgical Department of Lourenço Jorge Hospital - Rio de Janeiro - Brazil, Oral and Maxillofacial Surgery in private Clinic Grupo Face, Brazil

\section{Introduction}

The prosthetic rehabilitation of atrophic jaws has always been a challenge for the professionals involved in it. Depending on the degree of bone atrophy present not even the conventional prosthesis (dentures) cannot estore the aesthetics and function due to insufficient retention of the prosthesis, nerve compression pain, alteration of the proportions between the facial thirds and difficulties in the speech and Chewing. Due to increasing longevity, more active life and a growth in the elderly relationship in the percentage of the population, there has been an increase in the frequency of patients seeking the rehabilitation of the jaws for improving the quality of life.

The modern implant dentistry began in 1966 with the studies of Branemark precisely in this group of edentulous patients to enable aesthetic-functional rehabilitation through protocol type prostheses, where 6 implants were installed in the mandible and the patients received fixed prosthesis after 4 months. Branemark studies have demonstrated long-term predictable results being currently widely used for rehabilitation of these patients. The purpose of this article is to present a viable alternative to severely atrophied jaw rehabilitation with the use of osseointegrated implants and Branemark protocol prosthesis.

\section{Case Report}

Patient, N.S.A, 72 years old, was referred to the private practice with complaints of feeding and phonation difficulties due to the impossibility of using her inferior prosthesis. She said that at the age of 20 she no longer presented teeth in the mandible and maxilla due to socioeconomic problems that motivated the extraction of decayed elements. A Cone Bean computed tomography scan demonstrated severe bone atrophy with bilateral total inferior alveolar nerve exposure and mean available bone height at the mandibular symphysis of $4 \mathrm{~mm}$ (Figures $1 \& 2$ ). For the imminent risk of pathological fracture of the mandible, the planned treatment for the patient was the placement of four Cone Morse WS $(5.0 \times 5.0 \mathrm{~mm})$ implants (Neodent, Curitiba, Brazil) at the mandibular symphysis associated with grafting with Bio oss® (Geistilich Pharma AG, Switzerland) for gain of $1 \mathrm{~mm}$ in height.
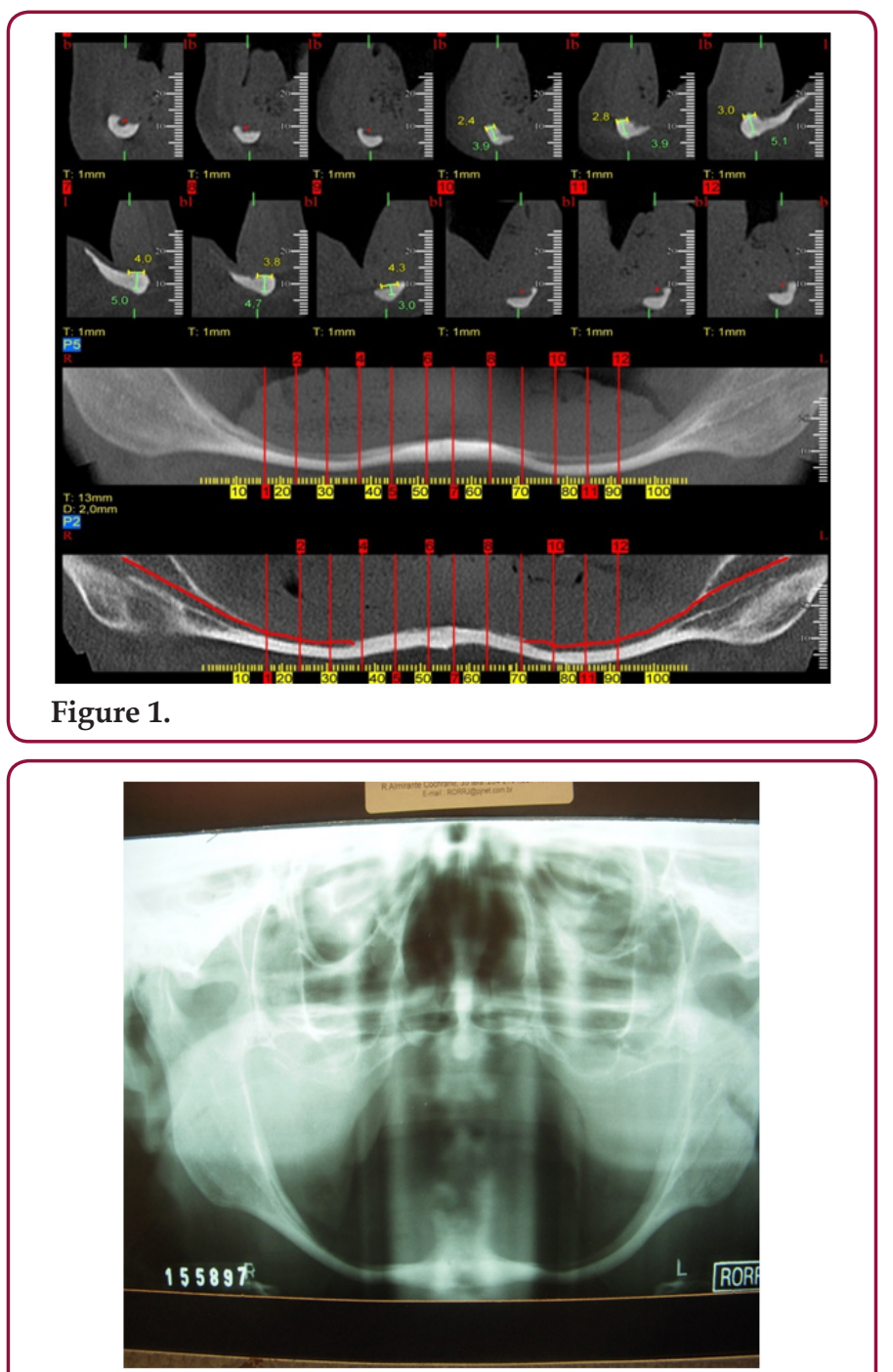

Figure 2. 
A Unilock® 2.0mm, 21-hole high profile (Synthes Maxillofacial, Paoli, PA) miniplate was used to strengthen the mandible using the area of the mandibular branches and mandibular symphysis for fixation (Figure 3). This plate was modeled prior to surgery with the aid of a biomodel made from the DICOM images of cone bean tomography. Pre-modeling of the miniplate allowed its insertion to be performed by trans-oral access, reducing the time and morbidity of the surgical procedure. After 4 months the creation of the Branemark protocol prosthesis type was started and the patient was extremely satisfied with the result obtained (Figures 4 \& 5). After 5 years of radiographic control we did not observe perimplant or any intercurrent bone loss (Figure 6).

Figure 3.
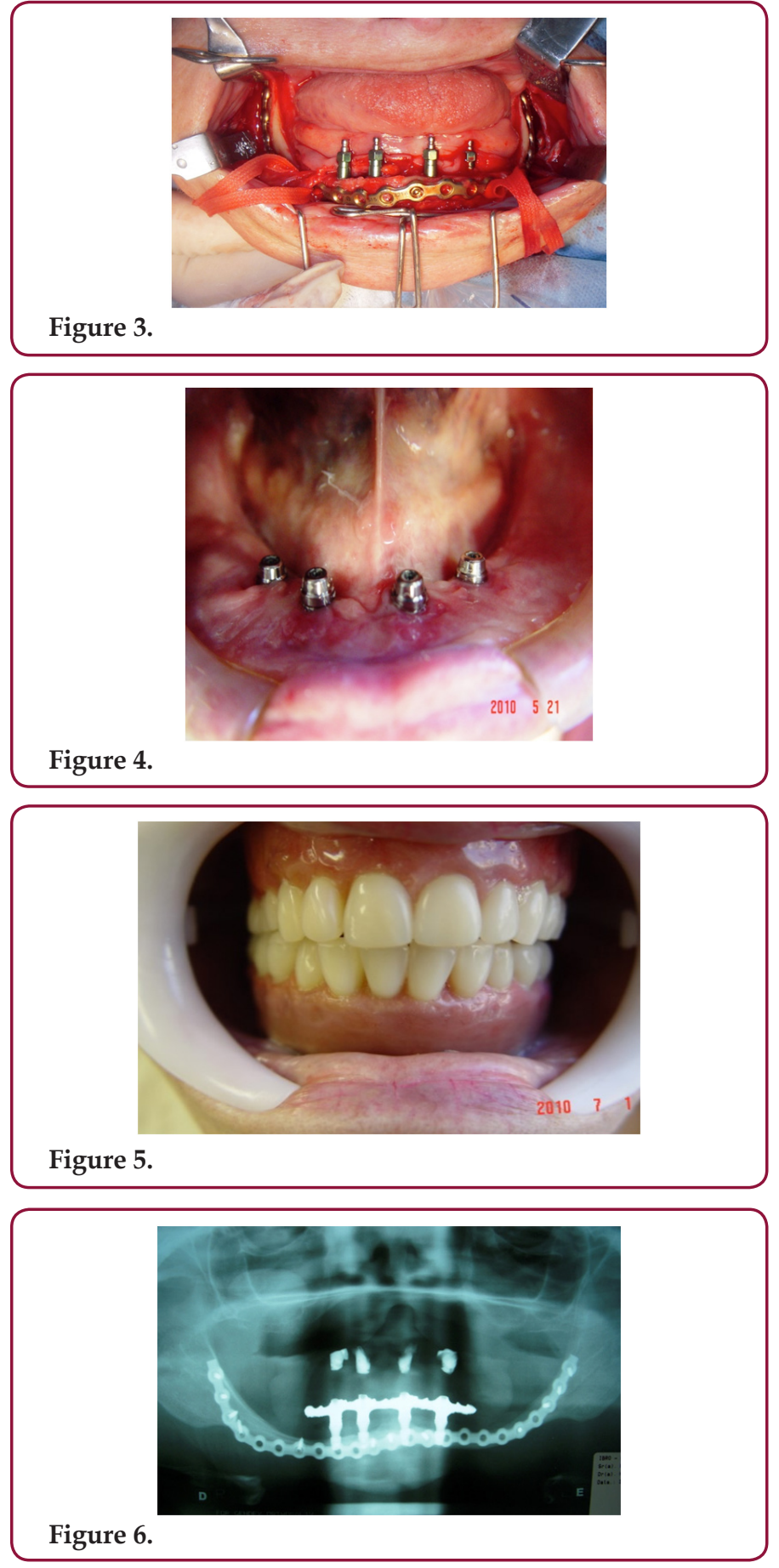

\section{Discussion}

The physiological changes associated with aging are factors that modify the physical, social and economic life of geriatric patients. These physiological changes may predispose or increase the susceptibility of elderly patients to dysfunction. The reduction of osteogenesis in elderly patients associated with loss of dental elements and consequent reabsorption of the alveolar bone are the main causes related to atrophy of the mandibular bone Bruce et al. [1]; Luhr et al. [2] developed a classification of the degree of mandibular atrophy with the purpose of establishing the level of difficulty in the treatment of fractures in atrophic mandibles. Jaws with $16-20 \mathrm{~mm}$ in height of remaining bone are classified as Class I.

Those with 11 to $15 \mathrm{~mm}$ of bone height remaining in Class II and finally those with less than $10 \mathrm{~mm}$ are classified as extremely atrophic or Class III. The classification of Cawood \& Howell [3] evaluates the degree of bone resorption from the moment the tooth was extracted. This classification ranged from 1 to 6 with 6 being the bone resorption end with the maintenance of the basilar bone alone. In the case in question the bone height in the anterior region of the mandible varied from 3.9 to $5 \mathrm{~mm}$ and in the region of mandible body was $2 \mathrm{~mm}$ demonstrating a residual bone extremely susceptible to pathological fracture of the mandible. In the past different surgical techniques have been proposed to allow the rehabilitation of atrophic jaws, including osteogenic distraction, onlay grafts, sandwich osteotomy, osteotomy, and graft in mandible basilar bone.

Many of these techniques presented predictable results, however, with increased morbidity of the surgical procedure and some of them with more than one surgical procedure to allow definitive prosthetic rehabilitation. In addition complications such as infection, loss of grafts and fracture of the mandible have been reported and are complications of difficult solution mainly in this group of patients. Bosker in 1996 developed the trans-mandibular implant system to be used on severely atrophied mandibles. Although the original article and subsequent articles have never defined the term "severely atrophied mandible", most patients included in these studies had residual bone in the anterior region of the mandible of $12 \mathrm{~mm}$ or less. The system consisted of a base plate that was attached to the basilar bone of the mandible that contained 4 trans-osseous pins where the prosthesis was made.

The disadvantage of this system is the need for extra-oral access for its insertion and the need for residual bone in the mandibular basilar of good quality for fixation of the anchor screws of the system. Some studies have shown success rates varying from 56\% to 75\% Bosker and VanDijk et al. [4-6]. In a study conducted in PubMed revealed 14 cases of atrophic mandible fractures secondary to the installation of implants in the anterior region in the period between 1990 and 2012. The authors excluded mandibular fractures secondary to lateralization of the inferior alveolar nerve associated with implant installation Almasri \& El-Hakin, Karlis [7]. In most reports the fracture occurred distally the installation of the implants. 
The first author to describe the technique used in this patient was Lopes et al. [8] combining concepts of treatment of atrophic mandible fractures and current concepts in Implantology, using a $2.0 \mathrm{~mm}$ system locking plate and 04 short implants that supported a Branemark protocol type prosthesis. Later, Lopes Alvarenga et al.[9] published a clinical case where they used a locking plate of the $2,4 \mathrm{~mm}$ system only in the anterior region of the mandible associated with 4 implants that supported an overdenture type prosthesis. The use of the locking plate in a severely atrophied mandible is justified by the necessity of support of the load bearing after the insertion of the prosthesis preventing the pathological fracture of the mandible. We understand that the region of the mandibular body is usually the region of greatest bone atrophy in extreme cases and due to the presence of the inferior alveolar nerve and inadequate bone height it is not always possible to use this region for plate fixation.

For this reason we understand that the plate has to be extended to the mandibular angle region bilaterally. In this case we chose to use $2.0 \mathrm{~mm}$ system plate because of the low bone height available for fixing the screws. In a randomized clinical study comparing 3 treatment modalities (trans mandibular implant, autogenous bone grafting for vertical gain followed by the installation of 4 implants and the installation of 4 short implants) of severely reabsorbed jaws, Stellingsma et al. [10] concluded that the use of short implants was the best modality of treatment due to the low rate of complications, high implants survival rate, stable implant-bone interface and the possibility of outpatient treatment. Freiberg et al. [11] demonstrated predictable long-term success rates with the use of short implants (Branemark implants 6-7mm) in mandibles without the use of bone grafts $(95.5 \%$ success rate at 5 years and $92.3 \%$ at 10 years of control). Deporter et al. [12] also demonstrated a $92.7 \%$ survival over 10 years with $0.03 \mathrm{~mm}$ mean annual bone loss in short implants that retained overdentures [13-14].

WS Neodent implants were chosen due to the lack of availability of short implants in the national market at the time of surgery. The Cone Morse platform can be easily modified with the placement of tapered minipillars during the prosthesis preparation. As a conclusion, this technique allows the rehabilitation of atrophic mandibles with a single surgical procedure and lower morbidity when compared with bone grafting procedures. A careful medical evaluation of the comorbidities present in this group of patients should be performed as well as the discussion about the advantages, disadvantages and possible complications.

\section{References}

1. Bruce AR, Ellis E (1993) the second Chalmers J. Lyons Academy study of fractures of the edentulous mandible. J Oral Maxillofac Surg 51(8): 904-911.

2. Luhr HG, Reidick T, Merten HA (1996) Results of tratment of fractures of the atrophic edentulous mandible by compression plating: a retospective evaluation of 84 consecutive cases. J Oral Maxillofac Surg 54(3): 250-254.

3. Cawood JI, Howell RA (1988) A classification of edentulous jaws. Int J Oral Maxillofac Surg 17(4): 232-236.

4. Bosker H, Van Dijk L (1989) the transmandibular implant: a 12-year follow-up study. J Oral Maxillofac Surg 47(5): 442-450.

5. Meijer HJ, Geertman ME, Raghoebar GM, Kwakman JM (2001) Implantretained mandibular overdentures: 6-year results of a multicenter clinical trial on 3 different implant systems. J Oral Maxillofac Surg 59(11): 1260-1268.

6. Paton G, Fuss J, Goss AN (2002) The transmandibular implant: a 5- and 15-year single-center study. J Oral Maxillofac Surg 60(8): 851-857.

7. M Almasri, M El-Hakim (2012) Fracture of the anterior segment of the atrophic mandible related to dental implants. International Journal of Oral \& Maxillofacial Surgery 41(5): 646-649.

8. Lopes, Oliveira DM, Vajgel A, Pita I, Bezerra T, et al. (2009) Reconstruction of Severely Atrophic Mandible. J Oral Maxillofac Surg 67(11):2455-2459.

9. Lopes Alvarenga, Rodrigo, Akaki Emilio, Rodrigues Antunes de Souza, Ana Cristina, et al. (2013) Reabilitação de mandibula atroficas com implantes curtos e placa de titanio: Apresentação de um caso clinico. Rev Port Estomaol Med Dent Cir Maxilofacac 54(4): 217-221.

10. Stellingsma K, Raghoebar GM, Meijer HJ, Stegenga B (2004) the extremely resorbed mandible: A comparative prospective study of 2 -year results with 3 treatment strategies. Int J Oral Maxillofac Implants 19(4): 563-577.

11. Friberg B, Gröndahl K, Lekholm U, Brånemark PI (2000) Long-term follow-up of severely atrophic edentulous mandibles reconstructed with short Brånemark implants. Clin Implant Dent Relat Res 2(4): 184-189.

12. Deporter D, Watson P, Pharoah M, Todescan R, Tomlinson G (2002) Tenyear of a prospective study using porous-surface dental implants and a mandibular overdenture. Clin Implant Dent Relat Res 4(4): 183-189.

13. Brånemark PI, Hansson BO, Adell R, Breine U, Lindstrom J, et al. (1977) Osseointegrated implants in the treatment of edentulous jaw. Experience from a 10-year period. Scand J Plast Reconstr Surg Suppl 16: 1-192.

14. Karlis V, Bae RD, Glickman RS (2003) Mandibular fracture as a complication of inferior alveolar nerve transposition and placement of endosseous implants: a case report. Implant Dent 12(3): 211-216.

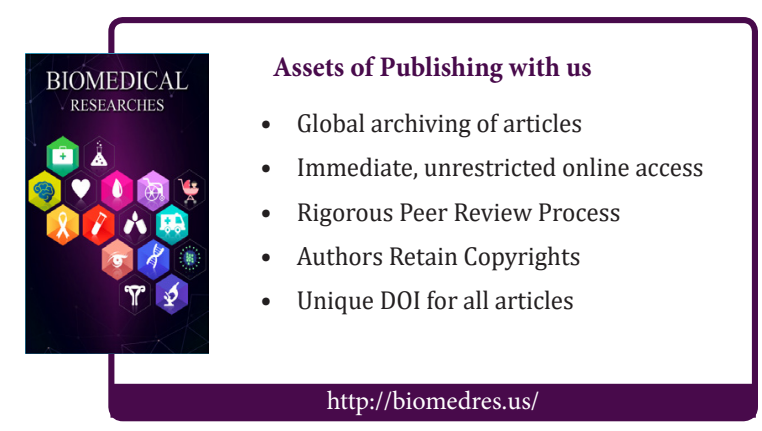

